\title{
Effect of Total Glucosides of Paeony on Imiquimod-Induced Psoriatic Skin Lesions by Regulating VEGF
}

\section{Yuan Zhang' \\ Xuan Zhou ${ }^{2}$ \\ Ledong Sun ${ }^{3}$}

'Department of Dermatology, Zhujiang Hospital, Southern Medical University, Guangzhou, 510000, People's Republic of China; ${ }^{2}$ Neonatal Intensive Care Unit, Zhujiang Hospital, Southern Medical University, Guangzhou, 510000, People's Republic of China; ${ }^{3}$ Department of Dermatology, The Fifth Affiliated Hospital, Southern Medical University, Guangzhou, People's Republic of China

Correspondence: Ledong Sun Department of Dermatology, The Fifth Affiliated Hospital, Southern Medical University, No. 566, CongCheng Road, Conghua District, Guangzhou, 510900,

People's Republic of China

Tel +86 I3829754I45

Email sunledong126@126.com
Purpose: The purpose of this study was to use a murine model of psoriasis to examine the effect of total glycosides of paeony (TGP) on psoriatic skin lesions and on the expression of vascular endothelial growth factor (VEGF) in skin lesions and blood.

Methods: A murine model of psoriasis was produced by shaving the backs of the mice and applying 5\% imiquimod cream, $50 \mathrm{mg}$, to the backs of the mice once a day. Mice were killed on day 8 , and skin and blood samples were obtained for histopathological examination and analysis of VEGF mRNA expression.

Results: By day 8 of the application of imiquimod cream, skin lesions characteristic of psoriasis were evident, and histopathological examination of skin sections showed changes consistent with psoriasis (corneum thickening and parakeratosis, attenuation of the stratum granulosum, thickening of the stratum spinosum, and lengthening of the epidermal ridge). In the treatment group, 7 days of treatment with TGP resulted in resolution of the skin lesions, and histopathological examination showed the epidermis and dermis are approximately normal, without corneum thickening, hyperkeratosis, and parakeratosis. On day 7 of treatment, skin expression of VEGF mRNA was significantly lower in the treatment group than in the group that did not receive treatment $(\mathrm{p}<0.05)$. Blood VEGF mRNA expression was not different between the groups.

Conclusion: TGP is effective for the treatment of psoriasis and may act by decreasing lesion VEGF mRNA expression.

Keywords: psoriasis, immune modulation, total glycosides of paeony, real-time fluorescent quantitative, RT-PCR, vascular endothelial growth factor, VEGF

\section{Introduction}

Psoriasis is a chronic inflammatory skin disease that affects $1 \%$ to $3 \%$ of the general population, and approximately 125 million people worldwide have psoriasis. ${ }^{1-4}$ The condition is characterized by abnormal epidermal dysplasia, infiltration of a large number of inflammatory cells in the dermis, and neovascularization. ${ }^{4-7}$ of the aforementioned histopathological features, abnormality of the dermal papillary vasculature appears first, suggesting that neovascularization is perhaps one of the key characteristics of psoriasis pathogenesis. ${ }^{8}$ However, the exact mechanisms of psoriasis pathogenesis remain unclear, although recent studies have suggested the interleukin (IL)-23/Th17 axis, and activation of innate immune cells and pathogenic T cells is likely involved. ${ }^{4,6,7}$

Vascular endothelial growth factor (VEGF) is a signaling protein that promotes the growth of new blood vessels, and one of the pathogenetic mechanisms in 
psoriasis is represented by the VEGF-induced angiogenesis. $^{5,9-12} \mathrm{VEGF}$ is markedly up-regulated in psoriatic skin lesions, ${ }^{9,13}$ and the psoriatic severity is correlated with the serum levels of VEGF. ${ }^{14}$ Moreover, single-nucleotide polymorphisms of the VEGF gene are associated with the pathogenesis of psoriasis. ${ }^{15,16}$

Total glucoside paeony (TGP) is an active compound extracted from the roots of Paeonia lactiflora Pall, and has been used as a traditional Chinese medicinal herb for thousands of years. ${ }^{17}$ It is used as an analgesic and antiinflammatory to treat conditions such as rheumatoid arthritis (RA). ${ }^{17}$ Recent studies have shown it to have immunomodulatory and antioxidant functions, and may be effective for the treatment of autoimmune conditions such as RA, oral lichen planus, and Sjögren syndrome. ${ }^{17}$ Study has also shown TGP to be beneficial for the treatment of psoriasis; ${ }^{17-19}$ however, the exact mechanisms of action are unclear. Moreover, whether VEGF is involved in the molecular mechanism of therapeutic effect of TGP on psoriasis remains to be investigated.

Thus, the purpose of this study was to use a murine model of psoriasis to examine the effect of TGP on psoriatic skin lesions and on the expression of VEGF in skin lesions and blood.

\section{Materials and Methods}

\section{Animal Experiments and Drug Treatment}

Sixty adult female BALB/c mice, weighing 18-20 g, were obtained from the Animal Experiment Center of Southern Medical University. The mice were randomly divided into 6 groups, with 10 mice in each group: 1) Treatment group; 2) Model group; 3) Control group; 4) Low-dose TGP group; 5) Middle-dose TGP group; 6) High-dose TGP group.

A murine model of psoriasis was produced by shaving the backs of the mice and applying 5\% imiquimod cream, $50 \mathrm{mg}$, to the backs of the mice once a day. ${ }^{20,21}$ All groups except for the Control group received treatment with imiquimod cream.

TGP (purity 90\%: Ningbo Langsheng Pharmaceutical Co., Ltd., China) was dissolved in normal saline to provide the dosages described below such that the defined dose was available in $0.4 \mathrm{~mL}$ of liquid. Mice in the low-, middle-, and high-dose groups were treated with $50 \mathrm{mg} /$ $\mathrm{kg}$ body weight TGP per day, $100 \mathrm{mg} / \mathrm{kg}$ TGP per day, and $200 \mathrm{mg} / \mathrm{kg}$ per day, respectively, by gavage. Mice in the Model group and Control group received $0.4 \mathrm{~mL}$ of normal saline by gavage daily. Mice in the Control group, Model group and the Low-, Middle-, and Highdose groups were killed on the 8th day and skin tissue and blood were collected for subsequent analysis. The mice in the Control group did not receive an application of imiquimod cream (only the back hair was removed).

For mice in the treatment group, 5\% imiquimod cream (50 mg/d) was applied on the backs (shaved) for 8 days. Beginning day 9, mice were treated with $100 \mathrm{mg} / \mathrm{kg}$ of TGP suspension by gavage once daily in addition to imiquimod cream daily. After 7 days of treatment, the skin lesions were resolved, the mice were killed, and skin tissue and blood were collected. Mice in the treatment group were treated with TGP until the skin lesions had resolved, which was to confirm that TGP does have a therapeutic effect on psoriasis. On the other hand, the mice in the low, middle-, and high-dose groups were killed when the skin lesions were significantly different among the 3 groups (Day 8), which was used to investigate an appropriate therapeutic concentration of TGP for psoriasis.

The experimental protocols were approved by the Institutional Review Board of Southern Medical University (NO. LAEC-2019-007). All animal experiments were conducted in accordance with standard Guidelines for the Care and Use of Laboratory Animals.

\section{Specimen Collection}

After the mice were killed, approximately $1 \mathrm{~mL}$ of whole blood was collected. The base skin on the backs of the mice was excised, treated and examined as described below.

\section{Histopathological Analysis of Skin Tissue}

Portions of the mouse skin tissue were fixed with a $4 \%$ formaldehyde solution for 15 hours, washed with running water for 2 hours, dehydrated with $50-100 \%$ concentration gradient ethanol, and then soaked in xylene for 2 hours. The sections were soaked in liquid paraffin in a $60^{\circ} \mathrm{C}$ incubator for 4 hours, and then embedded. The paraffin blocks were sliced into serial sections with a thickness of 5 $\mu \mathrm{m}$. The slices were deparaffinized by soaking in xylene for 20 minutes, followed by hydration with absolute ethanol $(95 \%, 85 \%, 75 \%)$ and distilled water for 2-5 minutes at each concentration, then stained with hematoxylin and eosin (HE). After embedding with the neutral resin, the slices were covered with coverslips and observed under a light microscope. In addition, the thickness of the 
epidermis was measured to determine thickening of the epidermis.

\section{Severity of Skin Lesions Assessment}

The severity of dorsal skin lesions was assessed by the psoriasis area and severity index (PASI). ${ }^{22}$ The erythema, scaling, and thickening were independently scored from 0 to 4 as follows: 0 , none; 1 , slight; 2 , moderate; 3 , marked; 4 , very marked. The cumulative score (erythema, scaly, thickening) is used as a measure of the severity of skin damage.

\section{Detection of VEGF mRNA in Skin Tissue and Peripheral Blood}

Real-time fluorescent quantitative (RT-PCR) was used to detect the expression of VEGF mRNA in the skin tissue and peripheral blood of each group of mice. In brief, tissue was placed into a mortar, a small amount of liquid nitrogen was added, and the tissue was quickly ground. Tissue total RNA was extracted using Trizol ${ }^{\circledR}$ according to the manufacturer's instructions. Peripheral blood total RNA was extracted using a Simply $\mathrm{P}$ Total RNA Extraction Kit (Hangzhou Bioer Technology Co., Ltd., China) according to the manufacturer's instructions. For reverse transcription, $2 \mu \mathrm{g}$ of total RNA was used as a template, and the first strand of cDNA was synthesized using a PrimeScript ${ }^{\circledR}$ 1st Strand cDNA Synthesis Kit (TAKARA, Japan). The cDNA template was used for fluorescence quantitative RT-PCR using a $\mathrm{SYBR}^{\circledR}$ Premix Ex Taq kit (TAKARA) on an Agilent Stratagene Mx3000P ${ }^{\circledR}$ Multiplex Quantitative PCR (QPCR) System to determine the expression levels of VEGF mRNA. GAPDH was used as the internal control, and data were analyzed by the $2^{-\Delta \Delta \mathrm{CT}}$ method.

\section{Statistical Analysis}

All outcomes were expressed as median and interquartile range (IQR: P25 to P75). Data were compared using the Wilcoxon rank-sum test. Correlation analysis was performed to determine correlations between the expression of VEGF mRNA in skin tissue and peripheral blood and the dosage of TGP given to the Low-, Middle-, and Highdoes groups. All statistical analyses were performed using SPSS version 17.0 software (SPSS Inc., USA). A value of $\mathrm{p}<0.05$ was considered to indicate statistical significance.

\section{Results General Condition of the Mice}

Except for the Control group, mice in the other groups that received treatment with $5 \%$ imiquimod cream gradually exhibited signs of fatigue, crouching, reduced food intake, and weight loss. However, there was no obvious hair loss, only the gloss of the hair decreased. At the time of application of the imquimod cream, the mice became agitated, resisted and became aggressive. One mouse in the Highdose group was killed by other mice in the same group.

In the Treatment group, by the 7th day of treatment the general condition of the mice had improved, their food intake had increased, and the skin lesions had resolved.

\section{Skin Changes}

Skin changes in the Control, Model, Low-, Middle-, and High-dose TGP groups on Day 4 and Day 8 are shown in Figure 1. In the Model group, 1 day after application of imiquimod cream red spots appeared on the skin; after 2-3 days, scales appeared; after 4-5 days, the red spots and scales worsened (Figure 1); after 6 days there was improvement in the severity of the skin lesions; At 7-8 days further deterioration and thickening was noted (Figure 1).

In the low-dose TGP group, after treatment on Day 8, the mice still had infiltrating erythema with a large number of scales, and the back hair could not be completely removed due to the adhesion of the skin rash and the hair (Figure 1). In the middle-dose TGP group, the mice's rash almost completely subsided, with only a few small scales (Day 8, Figure 1). In the high-dose TGP group, the mice showed light red patches with a few scales on the skin, but no obvious infiltration (Day 8, Figure 1).

In the Treatment group, after 7 days of treatment with TGP, the skin lesions resolved and the skin exhibited a normal appearance (Figure 2).

\section{Severity of Skin Lesions Assessment}

The severity of dorsal skin lesions was assessed by the PAS score. From day 3 to day 7, the Model group and the Lowdose, Middle-dose, and High-dose TGP groups had significantly higher total PASI score than the control group (all $\mathrm{P}<$ 0.001 , Figure 3), suggesting that the psoriasis model was successfully established. The total PASI score peaked on Day 5 or Day 6 in the Low-dose, Middle-dose, and Highdose TGP groups (Figure 3). On Day 7 and Day 8, the Lowdose, Middle-dose, and High-dose TGP groups had significantly lower total PASI score than the model group (all $\mathrm{P}<$ 

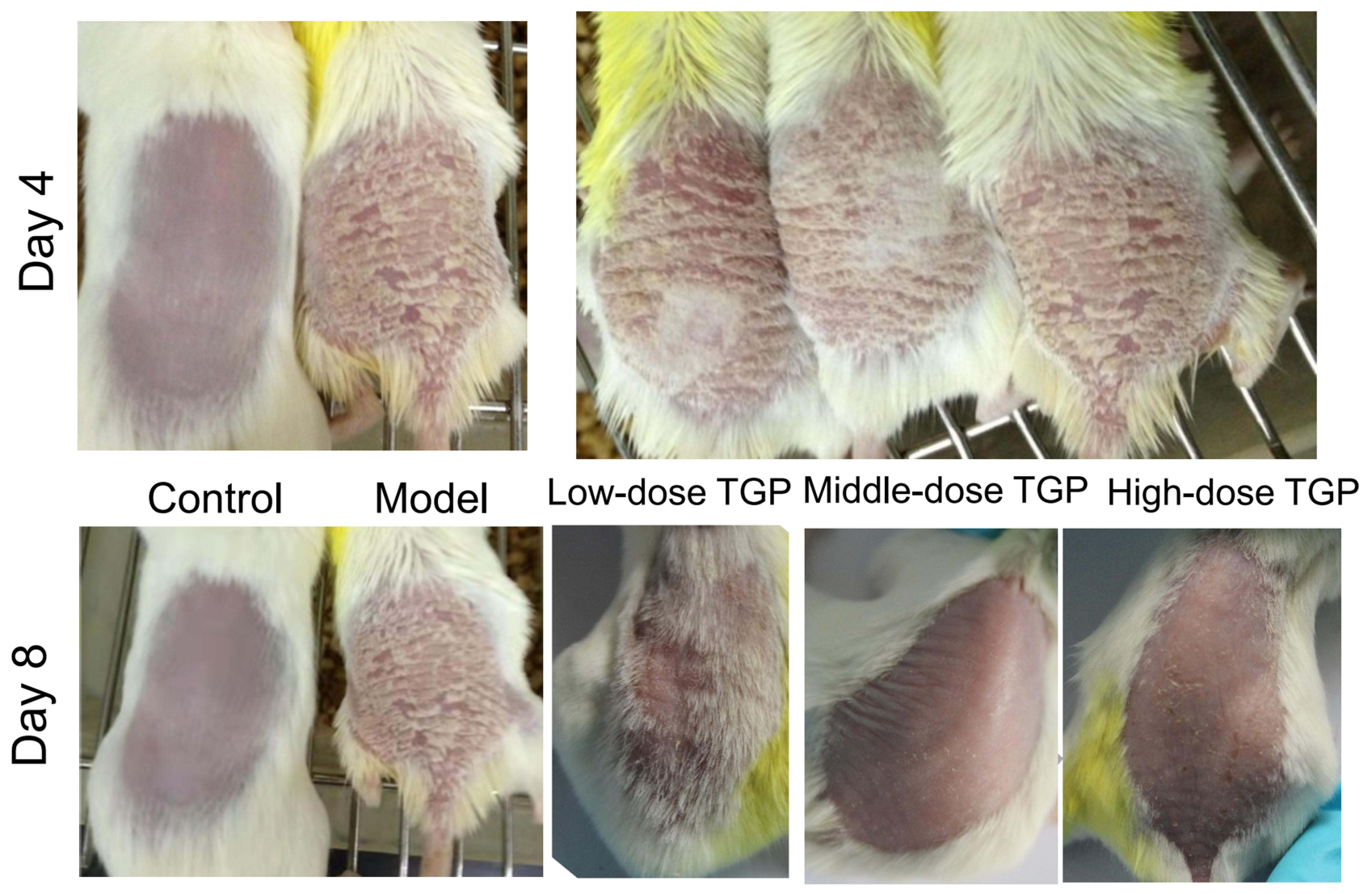

Figure I Skin changes in the Low-dose, Middle-dose, and High-dose TGP groups on Day 4 and Day 8.

0.001 , Figure 3 ), suggesting that TGP can effectively alleviate imiquimod-induced psoriatic skin lesions. There was no significant difference in total PASI score on Day 8 between the Middle-dose TGP group and the control group (Figure 3).

\section{Histological Examination}

In the Model group, histopathological examination of skin tissue on day 8 showed corneum thickening and parakeratosis, attenuation of the stratum granulosum, thickening of the stratum spinosum, and lengthening of the epidermal ridge (Figure 4). These histological changes are similar to those of human psoriasis.

In the Treatment group, histopathological examination showed the epidermis and dermis are approximately normal, without corneum thickening, hyperkeratosis, and parakeratosis (Figure 5).

\section{Effect of TGP on the Expression of VEGF mRNA in Skin Tissue}

Results of skin tissue VEGF mRNA expression are summarized in Figure 6. The VEGF mRNA expression levels in the Treatment group, the Middle- and High-dose groups, and the Control group were significantly lower than that of the Model group (all, $\mathrm{p}<0.05$ ). VEGF mRNA expression in the Low-dose TGP group was significantly higher than that of the Control group $(p=0.01)$, but not different from that of the Model group $(\mathrm{p}=0.32)$.

\section{Effect of TGP on the Expression of VEGF mRNA in Peripheral Blood}

Results of blood VEGF mRNA expression are summarized in Figure 6. The VEGF mRNA expression in the Model group was significantly higher than in the Control group $(p=0.00)$. However, the expression in the Model group was not different from that in any of the other groups (all, $\mathrm{p}>0.05$ ).

\section{Correlation Analysis}

A negative correlation between the concentration of TGP and the expression of VEGF mRNA in skin tissue was noted (Spearman's rho: $\mathrm{r}=-0.383, \mathrm{p}=0.040$, Figure 7A). However, the correlation was not significant $(r<0.50)$. 


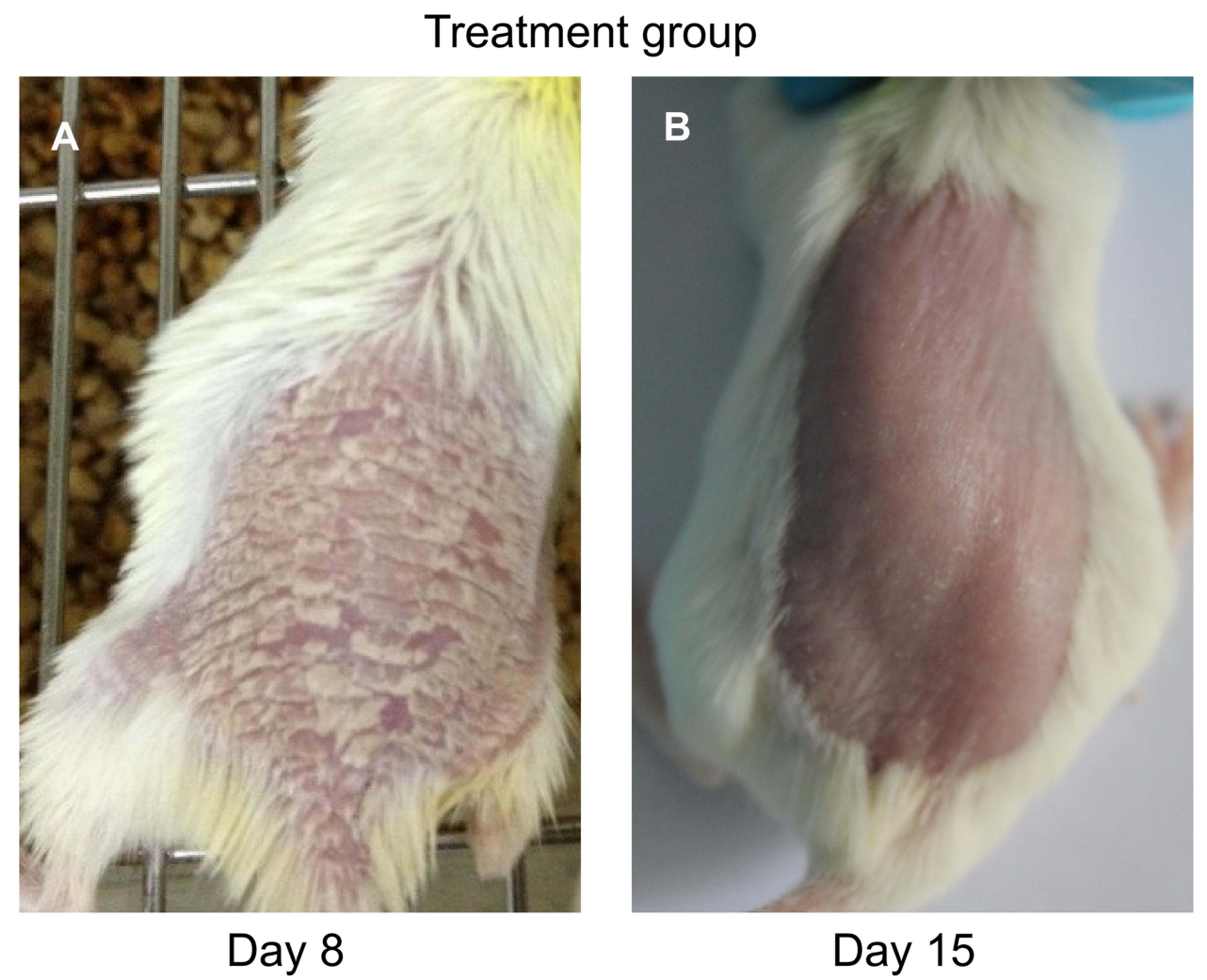

Figure 2 Images of skin of mice in the Treatment group (A) prior to treatment (Day 8) and (B) after treatment (Day 15). After 7 days of treatment with TGP the skin lesions have resolved and the skin appears normal (B).

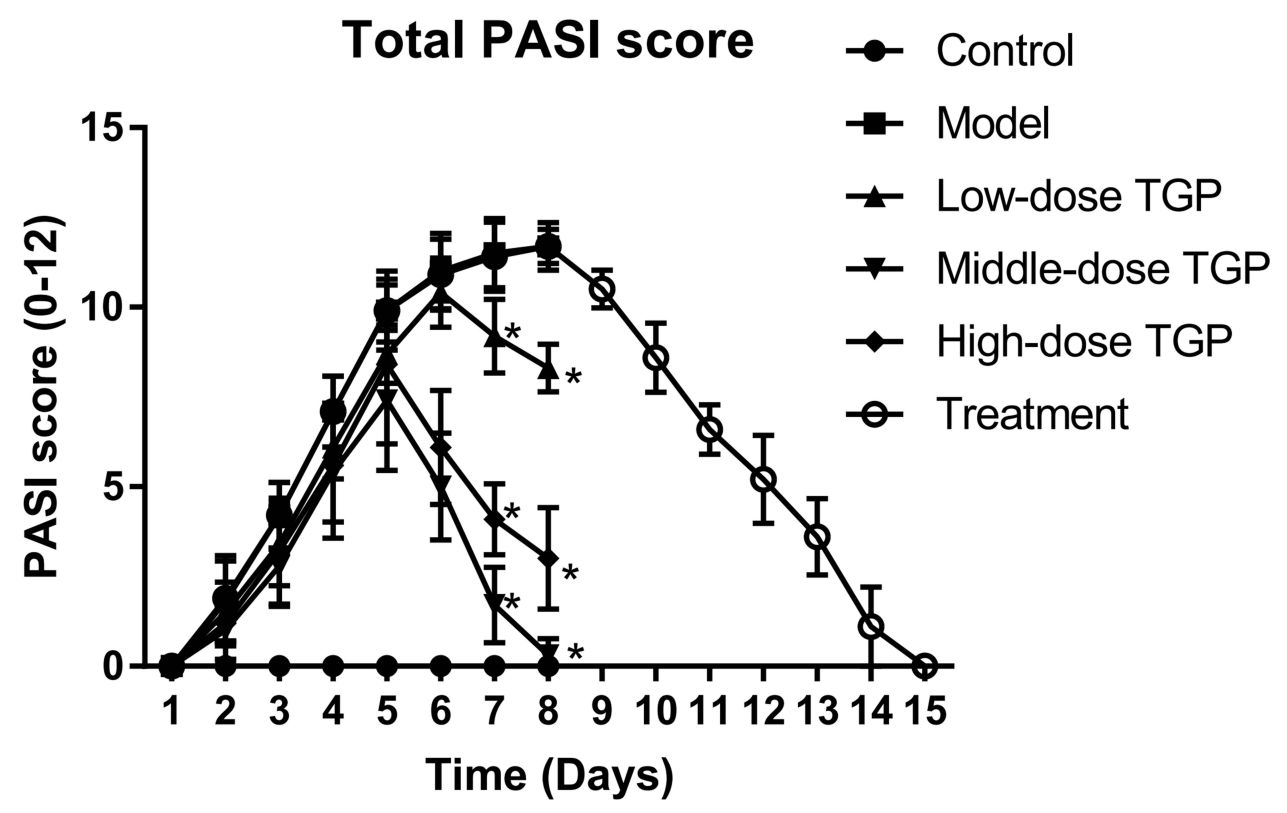

Figure 3 Psoriasis area severity assessment. The total PASI score was used to assess psoriasis area severity of skin lesions. Data are expressed as the mean \pm SD ( $=10$ for each group). $* \mathrm{P}<0.001$ vs the (untreated) Model group.

No correlation was noted between TGP concentration and the expression of VEGF mRNA in peripheral blood (Spearman's rho: $r=0.012, p=0.951$, Figure 7B).

\section{Discussion}

In this study, a murine model of psoriasis was developed by the topical application of $5 \%$ imiquimod cream. 

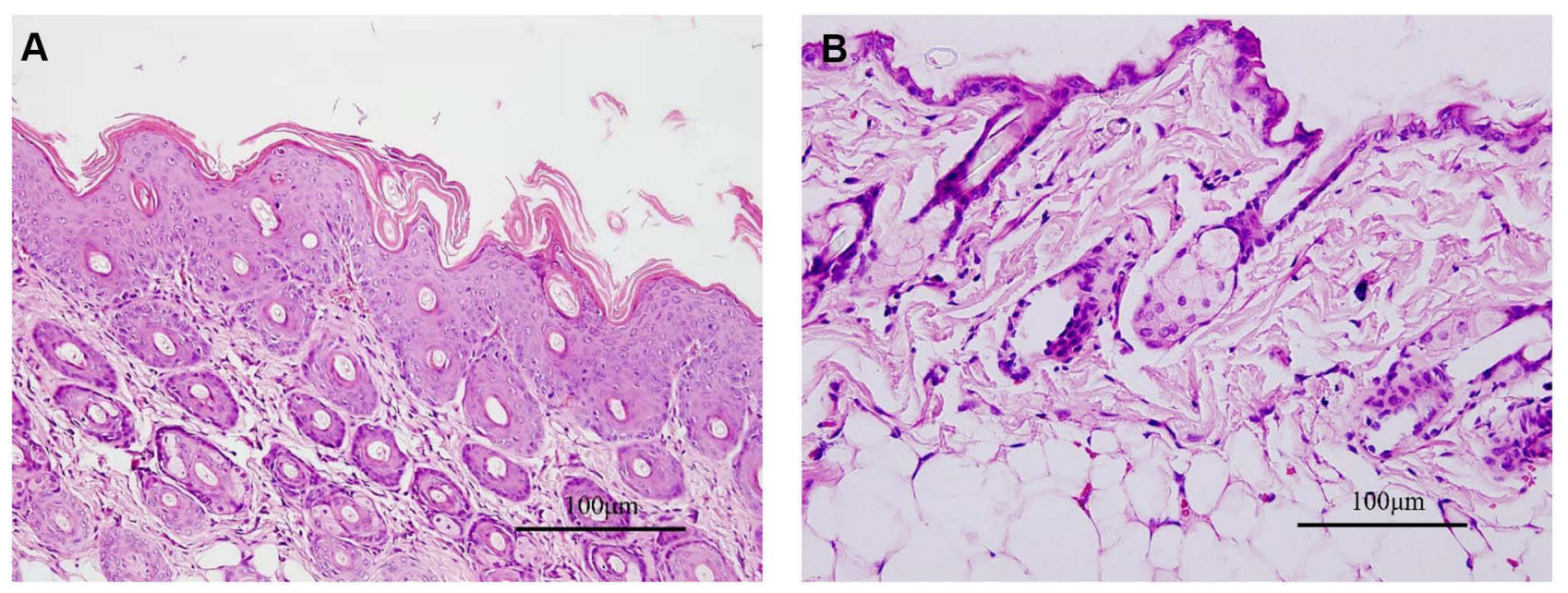

Figure 4 Histopathological examination of skin tissue of $(\mathbf{A})$ Model group and $(\mathbf{B})$ Control group on day 8 . Hematoxylin and eosin, scale bar= $100 \mu m$.
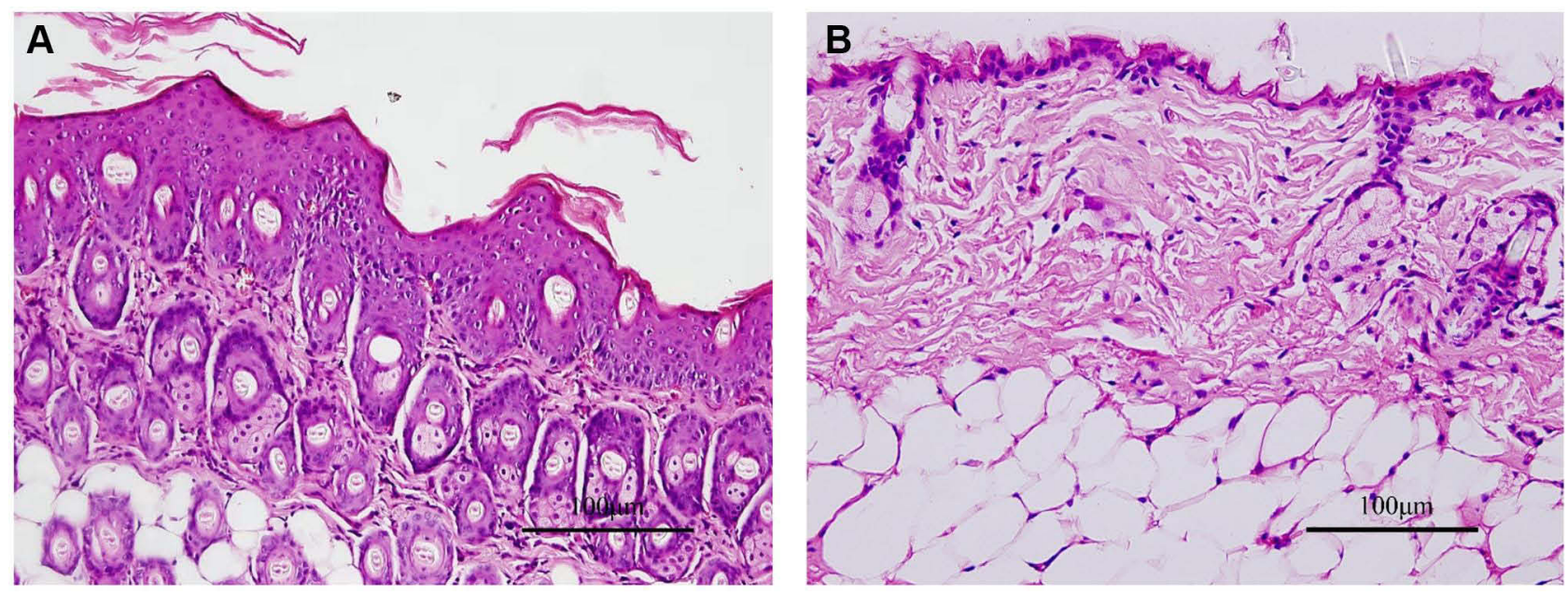

Figure 5 Histopathological examination of skin tissue of the Treatment group (A) prior to treatment and (B) after treatment. Hematoxylin and eosin (HE), scale bar $=100 \mu \mathrm{m}$. After 7 days of treatment with TGP light microscopy of HE stained tissue shows the epidermis and dermis are approximately normal, without corneum thickening, hyperkeratosis, and parakeratosis. (One of the mice in the Treatment group was killed on the 8th day to obtain skin tissue for histopathological examination. No other testing was performed on that mouse.).

Examination of the skin 8 days after beginning application showed characteristic lesions of psoriasis and characteristic histopathological changes. In addition, the expression of VEGF mRNA in skin tissue and peripheral blood of the Model group was significantly higher than that of Control group. This finding also showed, as seen in other studies, that high VEGF expression is related to the pathogenesis of psoriasis. ${ }^{9}$ Importantly, treatment with TGP for 7 days beginning on day 9 after the topical administration of imiquimod resulted in resolution of skin lesions and histopathological changes characteristic of psoriasis, and the VEGF mRNA levels were significantly decreased in the High-dose/Middle-dose TGP groups as compared with the model group. These results strongly suggest that middle and high dose of TGP is effective for the treatment of psoriasis. In contrast, VEGF mRNA expression was significantly higher in the Low-dose TGP group than in the Control group $(p=0.01)$. The skin of mice in the lowdose TGP group showed thickened erythema, a small number of white scales, and psoriasis lesions, suggesting low-dose TGP had no therapeutic effect on psoriasis. The correlation analysis also showed that the concentration of TGP was negatively correlated with VEGF mRNA the expression of in skin tissue. These results indicated that low-dose TGP had little therapeutic effect.

TGP is an immunomodulatory, and components include paeoniflorin, hydroxy-paeoniflorin, paeonin, albinorin, and benzoyl-paeoniflorin, with paeoniflorin 

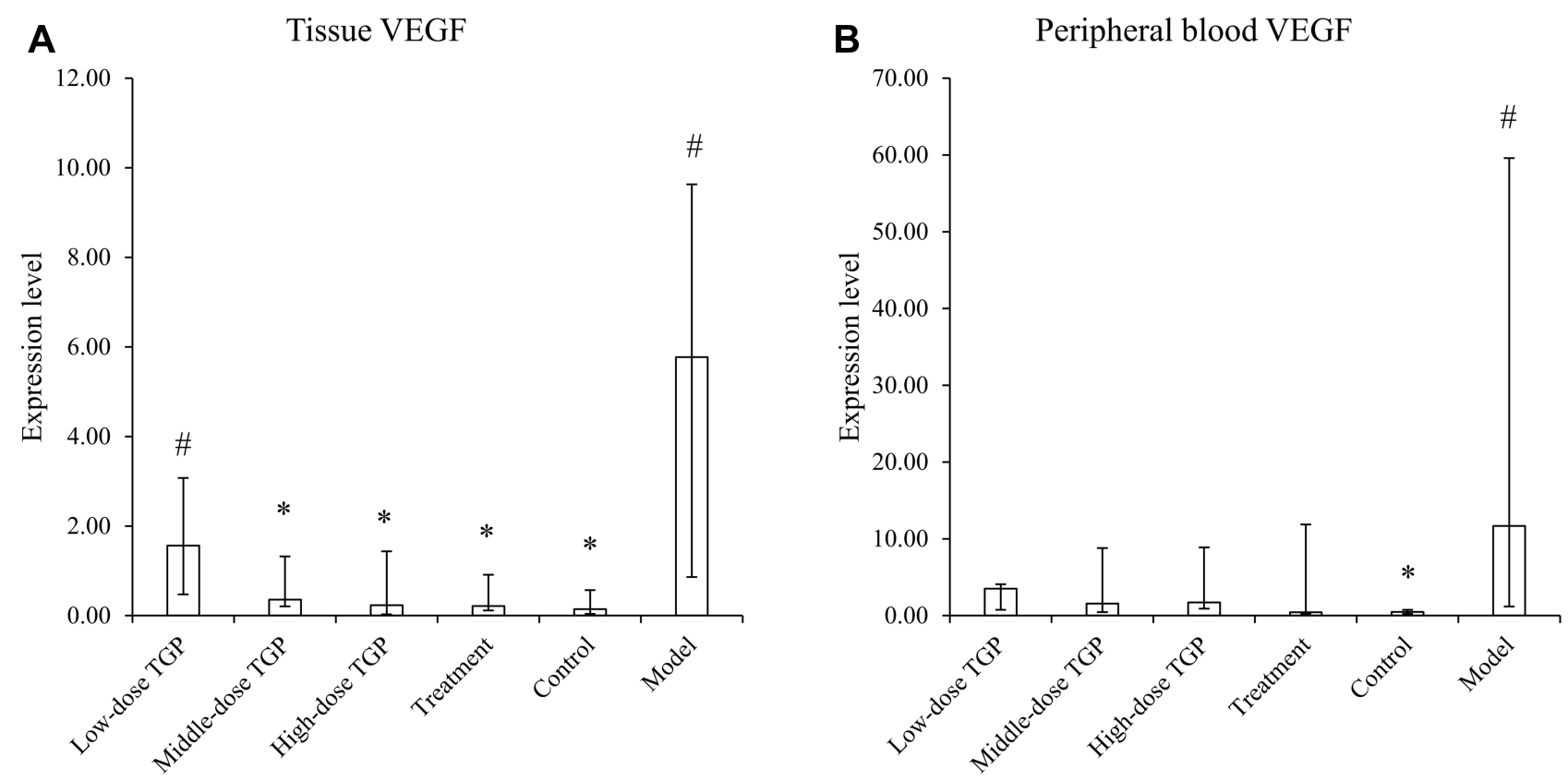

Figure 6 The mRNA expression level of skin tissue VEGF (A) and peripheral blood VEGF (B) among different groups. The bar chart statistics were median and IQR (interquartile range). ( $\mathrm{n}=10$ for each group) ${ }^{*} \mathrm{p}<0.05$ compared with the model group; ${ }^{*}<0.05$ compared with the Control group.
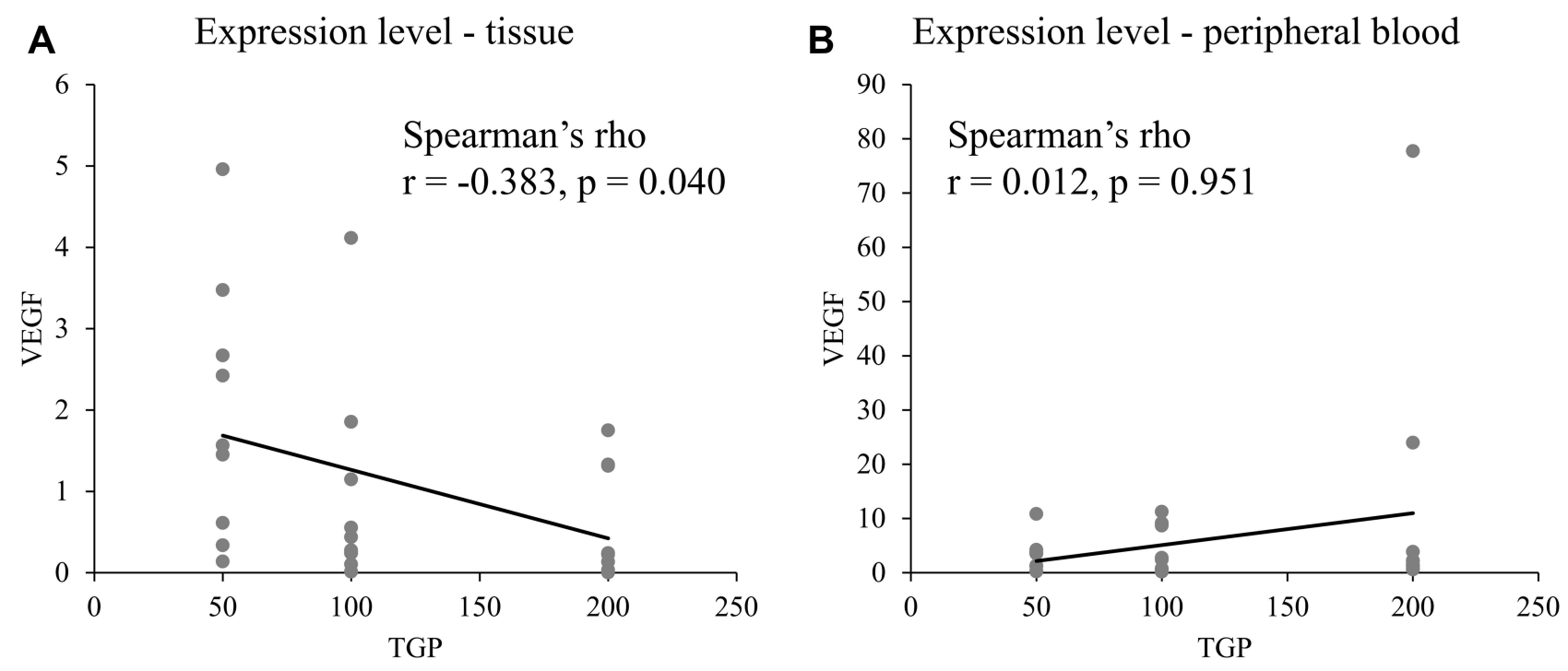

Figure 7 The $x-y$ scatter plot with regression line and Spearman's rho correlation coefficient between TGP and tissue VEGF expression level (A), TGP and peripheral blood VEGF expression level (B).

accounting for $90 \%$ of the total glycosides. ${ }^{23}$ TGP has been used for the treatment of autoimmune disorders, such as lichen planus, eczema, and systemic lupus erythematosus (SLE). ${ }^{17}$ Studies have also shown that TGP is beneficial for the treatment of psoriasis. ${ }^{18,19,24}$ A recent double-blind, randomized, placebo-controlled trial showed that TGP plus acitretin can enhance antipsoriatic efficacy and reduce liver damage as compared with acitretin treatment alone. ${ }^{19} \mathrm{~A}$ recent systematic review and meta-analysis of 30 randomized controlled trials that included almost 3000 patients concluded that the addition of TGP to other monotherapies for the treatment of psoriasis can improve the effective number and reduce psoriasis area and severity index (PASI), adverse reactions, recurrence rate, and inflammation reaction. ${ }^{18}$

Although TGP has been shown to be beneficial for treating psoriasis, the exact mechanisms of action have 
not been precisely revealed. Early studies have shown that pathological changes associated with psoriasis, such as neovascularization, are the result of overexpression of VEGF. ${ }^{12,25}$ It has also been shown that overexpression of VEGF promotes psoriatic pathological changes in the epidermis of mice; changes similar to those seen in human patients with psoriasis. ${ }^{9}$ Our results show that TGP has a therapeutic effect on psoriasis and it decreases the expression of VEGF mRNA in skin tissue, suggesting that the effect is a result of decreasing VEGF expression.

The p38 MAPK signal transduction pathway also plays a significant role in the pathogenesis of psoriasis, and P38 activity in psoriasis skin lesions is significantly higher than in the skin of unaffected, healthy individuals. ${ }^{26}$ P38 activity affects the release of many different downstream protein kinases and transcription factors, including VEGF, and over activation of $\mathrm{P} 38$ can cause an increase in VEGF expression. ${ }^{26}$ Prior study has also shown that TGP can inhibit the p38 MAPK pathway, ${ }^{17}$ suggesting that TGP has an anti-inflammatory effect and may affect VEGF expression by this mechanism.

Several limitations of this study should be pointed out. First, although we found VEGF may be involved in the molecular mechanism underlying the therapeutic efficacy of TGP on psoriasis, more involved signaling pathways remain to be elucidated. In addition, we did not set up a positive control group, as well as the corresponding control group and model group for the treatment group. Furthermore, the findings in the animal model should be validated in human patients as mouse model may not necessarily reflect the human model of psoriasis. All these limitations should be addressed in future studies.

\section{Conclusion}

The result of this study indicates that high to middle-dose TGP has a therapeutic effect on mice model of psoriasis, at least partially by inhibiting reducing VEGF expression. Nevertheless, it should be noted that mouse model may not necessarily reflect the human model of psoriasis.

\section{Abbreviations}

IL, interleukin; VEGF, vascular endothelial growth factor; TGP, total glucosides of paeony; RA, rheumatoid arthritis; HE, hematoxylin and eosin; PASI, psoriasis area and severity index; RT-PCR, real-time fluorescent quantitative.

\section{Data Sharing Statement}

All data generated or analyzed during this study are included in this published article.

\section{Ethics Approval and Informed Consent}

The experimental protocols were approved by the Institutional Review Board of Southern Medical University (NO. LAEC-2019-007). All animal experiments were conducted in accordance with standard Guidelines for the Care and Use of Laboratory Animals. Informed consent was not applicable.

\section{Funding}

There is no funding to report.

\section{Disclosure}

The authors report no conflicts of interest in this work.

\section{References}

1. Alex Anand D, Harishchander A, Jason UB. An integrated network analysis of psoriasis: a novel approach to disease pathology. Asian J Pharm Clin Res. 2015;8(3):176-178.

2. Bagchi S, Zhang H, He Y, Wang CR. Self-lipid specific T cells, restricted by $\mathrm{CD} 1 \mathrm{~b}$, contribute to the development of hyperlipidemia-associated skin inflammation (BA11P.133). J Immunol. 2015;194(1 Sup):184.15.

3. Gisondi P, Dalle Vedove C, Girolomoni G. Efficacy and safety of secukinumab in chronic plaque psoriasis and psoriatic arthritis therapy. Dermatol Ther. 2014;4(1):1-9. doi:10.1007/s13555-014$0042-5$

4. Armstrong AW, Read C. Pathophysiology, clinical presentation, and treatment of psoriasis: a review. $J$ Am Med Assoc. 2020;323 (19):1945-1960. doi:10.1001/jama.2020.4006

5. Costa C, Incio J, Soares R. Angiogenesis and chronic inflammation: cause or consequence? Angiogenesis. 2007;10(3):149-166. doi:10.1007/s10456-007-9074-0

6. Rendon A, Schäkel K. Psoriasis pathogenesis and treatment. Int J Mol Sci. 2019;20(6):1475. doi:10.3390/ijms20061475

7. Grän F, Kerstan A, Serfling E, Goebeler M, Muhammad K. Current developments in the immunology of psoriasis. Yale $J$ Biol Med. 2020;93(1):97-110.

8. Henno A, Blacher S, Lambert CA, et al. Histological and transcriptional study of angiogenesis and lymphangiogenesis in uninvolved skin, acute pinpoint lesions and established psoriasis plaques: an approach of vascular development chronology in psoriasis. J Dermatol Sci. 2010;57 (3):162-169. doi:10.1016/j.jdermsci.2009.12.006

9. Marina ME, Roman II, Constantin A-M, Mihu CM, Tătaru AD. VEGF involvement in psoriasis. Med Pharm Reports. 2015;88 (3):247-252. doi:10.15386/cjmed-494

10. Huggenberger R, Ullmann S, Proulx ST, Pytowski B, Alitalo K, Detmar M. Stimulation of lymphangiogenesis via VEGFR-3 inhibits chronic skin inflammation. J Exp Med. 2010;207(10):2255-2269. doi:10.1084/jem.20100559

11. Hvid H, Teige I, Kvist PH, Svensson L, Kemp K. TPA induction leads to a Th17-like response in transgenic K14/ VEGF mice: a novel in vivo screening model of psoriasis. Int Immunol. 2008;20 (8):1097-1106. doi:10.1093/intimm/dxn068

12. Yalçin B, Tezel GG, Arda N, Erman M, Alli N. Vascular endothelial growth factor, vascular endothelial growth factor receptor-3 and cyclooxygenase-2 expression in psoriasis. Anal Quant Cytol Histol. 2007;29(6):358-364. 
13. Dvorak AM, Feng D. The vesiculo-vacuolar organelle (VVO): a new endothelial cell permeability organelle. J Histochem Cytochem. 2001;49(4):419-432. doi:10.1177/002215540104900401

14. Nofal A, Al-Makhzangy I, Attwa E, Nassar A, Abdalmoati A. Vascular endothelial growth factor in psoriasis: an indicator of disease severity and control. J Eur Acad Dermatology Venereol. 2009;23 (7):803-806. doi:10.1111/j.1468-3083.2009.03181.x

15. Lee JH, Cho EY, Namkung JH, et al. Single-nucleotide polymorphisms and haplotypes in the VEGF receptor 3 gene and the haplotype GC in the VEGFA gene are associated with psoriasis in Koreans. $J \quad$ Invest Dermatol. 2008;128(6):1599-1603. doi:10.1038/sj. jid.5701204

16. Zablotna M, Sobjanek M, Nedoszytko B, et al. Association of psoriasis with the VEGF gene polymorphism in the northern Polish population. J Eur Acad Dermatology Venereol. 2013;27(3):319-323. doi:10.1111/j.1468-3083.2011.04393.x

17. Jiang H, Li J, Wang L, et al. Total glucosides of paeony: a review of its phytochemistry, role in autoimmune diseases, and mechanisms of action. J Ethnopharmacol. 2020;258:112913. doi:10.1016/j. jep.2020.112913

18. Zheng Q, Jiang WC, Sun XY, et al. Total glucosides of paeony for the treatment of psoriasis: a systematic review and meta-analysis of randomized controlled trials. Phytomedicine. 2019;62:152940. doi:10.1016/j.phymed.2019.152940

19. Yu C, Fan X, Li Z, Liu X, Wang G. Efficacy and safety of total glucosides of paeony combined with Acitretin in the treatment of moderate-to-severe plaque psoriasis: a Double-blind, randomised, placebo-controlled trial. Eur J Dermatol. 2017;27(2):150-154. doi:10.1684/ejd.2016.2946
20. Xia YP, Li B, Hylton D, Detmar M, Yancopoulos GD, Rudge JS. Transgenic delivery of VEGF to mouse skin leads to an inflammatory condition resembling human psoriasis. Blood. 2003;102(1):161-168. doi:10.1182/blood-2002-12-3793

21. Li B, He S, Liu R, et al. Total glucosides of paeony attenuates animal psoriasis induced inflammatory response through inhibiting STAT1 and STAT3 phosphorylation. J Ethnopharmacol. 2019;243:112121. doi:10.1016/j.jep.2019.112121

22. Li XQ, Chen Y, Dai GC, Zhou BB, Yan XN, Tan RX. Abietic acid ameliorates psoriasis-like inflammation and modulates gut microbiota in mice. J Ethnopharmacol. 2021;272:113934.

23. Zhang L, Wei W. Anti-inflammatory and immunoregulatory effects of paeoniflorin and total glucosides of paeony. Pharmacol Ther. 2020;207:107452. doi:10.1016/j.pharmthera.2019.107452

24. Guan XNW. Total glucosides of Paeonia's influence on T-cell subgroup in peripheral blood of common plaque psoriasis. Henan Tradit Chinese Med. 2010;30:455-456.

25. Simonetti O, Lucarini G, Goteri G, et al. VEGF is likely a key factor in the link between inflammation and angiogenesis in psoriasis: results of an immunohistochemical study. Int $J$ Immunopathol Pharmacol. 2006;19(4):751-760. doi:10.1177/039463200601900405

26. Mavropoulos A, Rigopoulou EI, Liaskos C, Bogdanos DP, Sakkas LI. The role of p38 mapk in the aetiopathogenesis of psoriasis and psoriatic arthritis. Clin Dev Immunol. 2013;2013:569751. doi: $10.1155 / 2013 / 569751$

\section{Publish your work in this journal}

Clinical, Cosmetic and Investigational Dermatology is an international, peer-reviewed, open access, online journal that focuses on the latest clinical and experimental research in all aspects of skin disease and cosmetic interventions. This journal is indexed on CAS.
The manuscript management system is completely online and includes a very quick and fair peer-review system, which is all easy to use. Visit http://www.dovepress.com/testimonials.php to read real quotes from published authors. 